\title{
Different effects of agroclimatic factors on time to emergence and time to flowering in nine soybean accessions
}

\author{
K. Kozlov ${ }^{*}$, L. Novikova ${ }^{1,2}$, I. Seferova ${ }^{2}$, S. Nuzhdin ${ }^{1,3}$, M. Samsonova ${ }^{1}$ \\ ${ }^{1}$ Peter the Great St.Petersburg Polytechnic University, St.Petersburg, Russia \\ ${ }^{2}$ Federal Research Center the N.I. Vavilov All-Russian Institute of Plant Genetic Resources, \\ St. Petersburg, Russia \\ ${ }^{3}$ University of Southern California, Los Angeles, CA, USA \\ *e-mail:kozlov_kn@spbstu.ru
}

Key words: soybean, time to emergence, time to flowering, agroclimatic factors, grammatical evolution

Motivation and Aim: The effect of climate change on agronomically important crops can be assessed with regression models that connect agronomic traits to climatic factors. Accounting for interactions between genotype and environment will provide valuable insights into phenological characteristics of cultivars across different geographical locations and genotypes.

Methods and Algorithms: We build a regression model for time periods from sowing to emergence and emergence to flowering as a linear combination of $N$ control functions $F_{n}$ that describe the dependence of phenotype on climatic factors. The interaction between genotype and environment is modeled by additional term in regression function that has the form of a weighted sum of pairwise products between a control function and each accession. We use a combination of Grammatical Evolution, LASSO and Differential Evolution [1] to recover analytic form of control functions, find regression coefficients and determine the set of significant climatic factors, respectively. Due to a stochastic nature of the procedure it is repeated several times to obtain an ensemble of models. Consequently, the effect of a factor on accession is estimated with the coefficient of determination averaged over the ensemble of models, from which the terms that do not depend on the factor were excluded. To reveal factors that affect accessions differently, their effects on accession pairs were compared with Wilcoxon test.

Results: The method was applied to predict the time periods from sowing to emergence (coefficient of determination $R^{2}=0.65$ ) and from emergence to flowering $R^{2}=0.65$ ) in a dataset that comprises 379 plants of 9 soybean accessions of different origin phenotyped at Pushkin and Kuban VIR stations in 1999-2013 [2].

Conclusion: Our analysis allowed us to reveal pairs of accessions with quantitatively different reaction on temperature and precipitation. The analysis of relative difference in the sum of squares for a model with and without a weighted sum of pairwise products between a control function and each accession allows us to conclude that genotypeenvironment interaction accounts for about $6.3 \%$ and $15.9 \%$ of variation in time periods from sowing to emergence and emergence to flowering, respectvely.

Acknowledgements: Supported by the Federal Targeted Program (Agreement No. 14.575.21.0136). Calculations were performed in Supercomputer Center of Peter the Great St.Petersburg Polytechnic University and University of Southern California CBB computer cluster.

\section{References}

1. Kozlov K. et al. (2018) A Mathematical Model of the Impact of Climatic Factors on Soybean Development. Biophysics. 63(1):175-176.

2. Seferova I., Novikova L. (2015) Climatic factors that impact development of fast ripening accessions of soybean in Nothwest Russia. Applied Botany, Genetics and Selection. 176:88-97. 